\title{
Diagnosing Pathological Prognostic Factors in Retinoblastoma: Correlation between Traditional Microscopy and Digital Slides
}

\author{
Pablo Zoroquiain ${ }^{a, b}$ Patrick Logan ${ }^{a}$ Vasco Bravo-Filho ${ }^{a}$ Natalia Vila $^{a}$ \\ Samir Jabbour ${ }^{a}$ Maria Eugenia Orellana ${ }^{a}$ Miguel N. Burnier ${ }^{a}$ \\ ${ }^{a}$ Henry C. Witelson Ocular Pathology Laboratory, McGill University, Montreal, Que., Canada; \\ ${ }^{b}$ Department of Pathology, School of Medicine, Pontificia Universidad Catolica de Chile, \\ Santiago, Chile
}

\section{Key Words}

Virtual slides · Whole slide images · Retinoblastoma · Ocular pathology

\begin{abstract}
Objective: It was the aim of this study to determine the diagnostic accuracy of high-risk prognostic factors and morphological characteristics of retinoblastomas using digital whole slide images (WSI) generated by a scanner. Methods: Forty-seven H\&E sections of glass slides with high-risk morphological features of retinoblastoma were analyzed. Slides were scanned as WSI and reviewed. The results were compared with those obtained after reviewing the slides using a regular microscope as the gold standard. McNemar's test (MT), the percentage of agreement (POA), and sensitivity (S) and specificity (Sp) were evaluated between WSI and conventional microscopy. Results: There were no differences with respect to multicentricity, growth type, rosette formation, choroidal invasion, anterior chamber invasion, extraocular extension, scleral extension, optic nerve invasion, necrosis, or Azzopardi effect between WSI analysis and light microscopy (MT, $p=1.0 ; \mathrm{POA}=100 \%$; $\mathrm{S}=100 \%$, and $\mathrm{Sp}=100 \%$ ). Discordance was found in 1 case where calcification could not be found using WSI (MT, $p=1.00 ; \mathrm{POA}=97.9 \% ; \mathrm{S}=100 \%$, and $\mathrm{Sp}=97.8 \%$ ). Conclusion: To the best of our knowledge, this is the first report using digital pathology (WSI) to evaluate prognostic factors in eyes containing retinoblastomas. Using WSI, the pathologist was able to detect highrisk morphological features in retinoblastoma. To date, WSI is an important tool, in particular for ophthalmic pathologists examining enucleation and exenteration specimens.
\end{abstract}

(C) 2015 S. Karger AG, Basel 


\section{Ocular Oncology \\ and Pathology}

\begin{tabular}{l|l|}
\hline Ocul Oncol Pathol 2015;1:259-265 \\
\hline DOI: 10.1159/000381155 & $\begin{array}{l}\text { (c) 2015 S. Karger AG, Basel } \\
\text { www.karger.com/oop }\end{array}$ \\
\hline
\end{tabular}

Zoroquiain et al.: Diagnosing Pathological Prognostic Factors in Retinoblastoma: Correlation between Traditional Microscopy and Digital Slides

\section{Introduction}

The development and application of the whole slide imaging (WSI) technology has had a significant impact on digital imaging in pathology $[1,2]$. WSI is the reduction of a microscope slide to a digital image that is viewed on a computer screen rather than through a microscope [2]. Typically, the image is captured at $20 \times$ or $40 \times$ magnifications; however, using software, the user can view the image at different 'magnifications' at the desired level on a computer screen (e.g., $4 \times, 10 \times, 20 \times$, or $40 \times$ ).

WSI technology has several advantages over conventional microscopy, such as portability (i.e., images are accessible anywhere and at any time without the risk of being broken or lost), the ability to easily share images with specific annotations in areas of interest, to conduct a remote conference meeting with real-time visualization, and also to make use of computer-aided diagnostic tools (e.g., image analysis) [1].

Despite the fact that digital imaging in pathology is not accessible to most pathologists at present, it has been used extensively by students and at conferences for some time; however, its use as a diagnostic tool is still controversial. Recently, a bulletin by the College of American Pathologists offered guidelines in order to validate WSI as a diagnostic tool [1]. There are even a few WSI providers that have obtained a class II license for diagnosis [3], thereby spring-boarding pathologists into a new era of digital diagnostic pathology.

WSI in ocular pathology not only supports triaging of difficult cases among specialists within an organization, but it also enables remote pathology consultations with external organizations around the world [4]. In order to analyze and compare WSI to traditional microscope-based pathology, we reviewed retinoblastoma (RB) cases. RB is the most common primary intraocular malignant tumor in children, and it represents approximately $2.5 \%$ of cancer cases in this age group [5]. Accurate pathological analysis after enucleation is vital to define prognosis, follow-up, and future patient treatments [6]. Currently, all enucleation specimens are evaluated using conventional glass microscopy, and the reports are generated according to the 2009 International Retinoblastoma Staging Working Group [6]. The most important prognostic factor in RB is the infiltration of the optic nerve; this infiltration can be prelaminar, laminar, or retrolaminar, all of which impart a different prognosis [6]. Finally, RB was selected for our analysis due to these numerous prognostically relevant structures which have different characteristics at different magnifications and represent a real challenge for ocular pathologists.

To the best of our knowledge, this is the first report of WSI applied in ophthalmic pathology. The aim of the present study was to compare the accuracy of using WSI digital pathology as a tool for the pathologist to detect high-risk morphological features and other morphological aspects in RB samples with conventional microscopy.

\section{Methods}

In order to identify high-risk morphological features of RB, 128 optopupillary (OP) sections of RB glass slides stained routinely with H\&E were reviewed. Forty-seven cases that had at least one morphological risk factor were collected from the Henry C. Witelson Ocular Pathology Laboratory, Montreal, Que., Canada. They were further analyzed as WSI obtained with iScan Coreo (Ventana, Tucson, Ariz., USA ) at 40× magnification and reviewed using the Virtuoso ${ }^{\mathrm{TM}}$ image analyzer (Ventana). The following morphological features were examined: presence of multifocal tumor (unique/multiple), type of growth (endophytic/exophytic/diffuse), differentiation of the tumor estimated as the presence and type of rosette formation (Homer Wright, FlexnerWintersteiner, and fleurettes), choroidal invasion [focal $(<3 \mathrm{~mm}) /$ massive $(>3 \mathrm{~mm}$ ] [6], invasion of anterior chamber structures, extraocular extension, extension to the sclera (including vortex vessels), optic nerve invasion (head or prelaminar, laminar, and postlaminar invasion), necrosis, calcification, and Azzopardi effect. 


\section{Ocular Oncology \\ and Pathology}

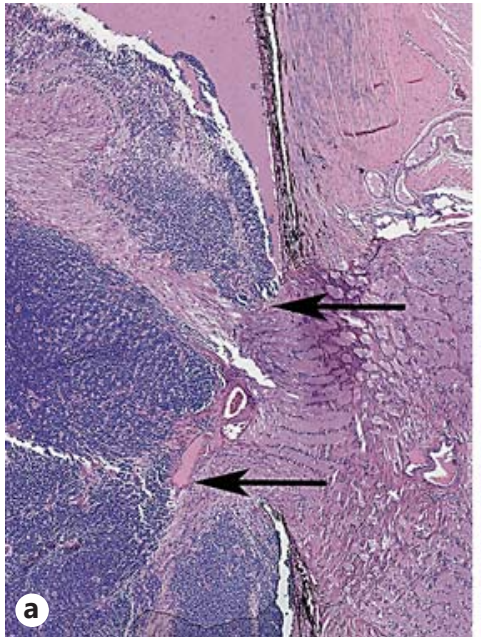

Zoroquiain et al.: Diagnosing Pathological Prognostic Factors in Retinoblastoma: Correlation between Traditional Microscopy and Digital Slides
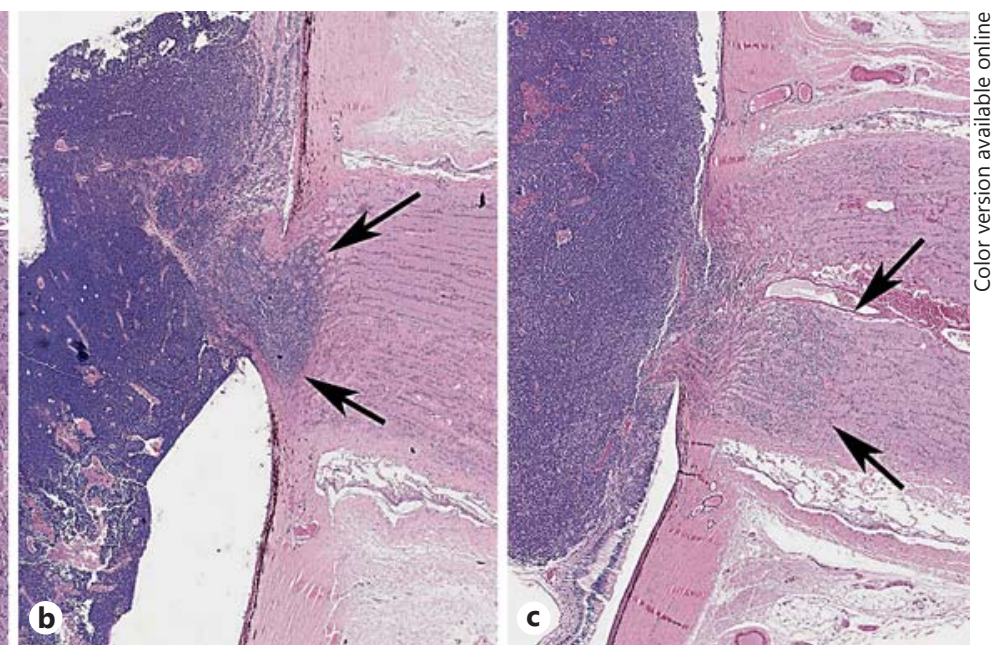

Fig. 1. a RB infiltrating the optic nerve. $\mathbf{b}$ RB with infiltration up to the lamina cribosa. $\mathbf{c}$ RB showing infiltration into the optic nerve. The arrows indicate the deepest infiltration.

\section{Whole Slide Imaging}

Briefly, glass pathology slides were loaded into the scanner (up to 160 slides at a time) and were continuously scanned at high speed under a microscope objective lens. As the histopathology specimen has tissue sections of known thickness, real-time focus adjustments determined by software algorithms were utilized to keep the resulting images in focus. The objective lens determines the output resolution of the images and also the overall speed of capture, with a higher resolution resulting in smaller fields of view and longer scan times [4]. The resulting image was represented in 24-bit real color with dimensions of thousands of pixels in both width and height. Due to the massive amount of data obtained, the image was stored in a pyramid-like structure organized into layers, each of a different resolution, to facilitate efficient viewing via the Virtuoso image analyzer.

\section{Statistical Methods}

All analyses were performed using the Statistical Package for the Social Sciences 15.0 (SPSS Inc., Chicago, Ill., USA). In order to assess correlations of morphological features between WSI and conventional microscopy, McNemar's test (MT), percentage of agreement (POA), sensibility (S), and specificity (Sp) were determined. A $\chi^{2}$ test was performed in order to assess the correlation for invasion of the optic nerve between WSI and conventional microscopy.

\section{Results}

\section{Morphological Risk Factors}

Optic nerve invasion was detected in 44 cases $(93.6 \%)$, and the correlation between viewing methods was perfect (MT, $\mathrm{p}=1.00 ; \mathrm{POA}=100 \% ; \mathrm{S}=100 \%$, and $\mathrm{Sp}=100 \%)$. The types of invasion included prelaminar (36.4\%; fig. 1a), laminar (15.9\%; fig. 1b), and postlaminar (47.7\%; fig. 1c), with a POA of $100 \%$ and a $\chi^{2}$ of 1.00 .

Choroidal invasion was present in 25 cases (53.2\%), which was graded as focal in 8 (17.0\%; fig. 2b) and massive in 17 cases (38.6\%; fig. 2c). Extraocular spread was present in 7 cases (14.9\%; fig. 3a), intrascleral invasion was detected in 12 cases $(25.53 \%)$, and anterior chamber invasion was present in 12 cases (25.5\%; fig. 3b). All these risk factor were compared between WSI and conventional microscopy analyses, and no differences were detected $(\mathrm{MT}, \mathrm{p}=1.00 ; \mathrm{POA}=100 \% ; \mathrm{S}=100 \%$, and $\mathrm{Sp}=100 \%$ ). 


\section{Ocular Oncology and Pathology}

\begin{tabular}{l|l}
\hline Ocul Oncol Pathol 2015;1:259-265 \\
\hline DOI: 10.1159/000381155 & $\begin{array}{l}\text { @ } 2015 \text { S. Karger AG, Basel } \\
\text { www.karger.com/oop }\end{array}$ \\
\hline
\end{tabular}

Zoroquiain et al.: Diagnosing Pathological Prognostic Factors in Retinoblastoma: Correlation between Traditional Microscopy and Digital Slides
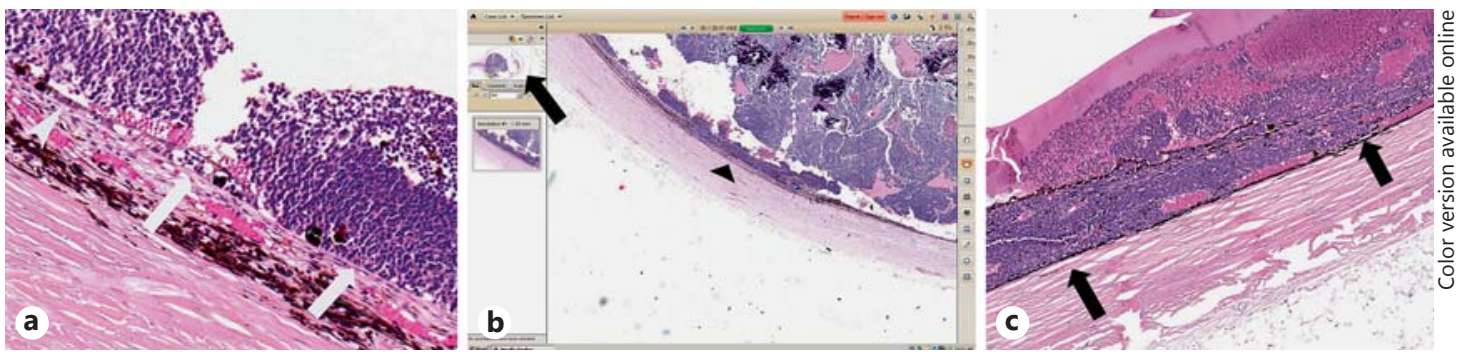

Fig. 2. a RB without choroidal invasion. The neoplastic cells are above the retinal pigmented epithelial cells (arrowhead), disrupting them (broad arrow) and pushing Bruch's membrane but without infiltration (small arrow). $\mathbf{b}$ Virtuoso view showing the whole specimen as a navigation window (arrow). In the user window, an RB with focal choroidal infiltration measuring $1.5 \mathrm{~mm}$ (arrowhead) is shown; the blue line represents the measurement tool. c Massive choroidal infiltration occupying the choroid and pushing the sclera (arrows).

Fig. 3. a RB with massive extraocular extension. $\mathbf{b}$ The RB is infiltrating the iris (large arrow) and the ciliary body (arrowhead). Vitreal seeding is also observed above the ciliary body epithelium (small arrow). cWell-differentiated RB with calcifications. d Deposits of basophil pigment in the walls of blood vessels known as Azzopardi effect.

Fig. 4. a Well-differentiated RB with Homer Wright rosettes. b Well-differentiated RB with Flexner-Wintersteiner rosettes.
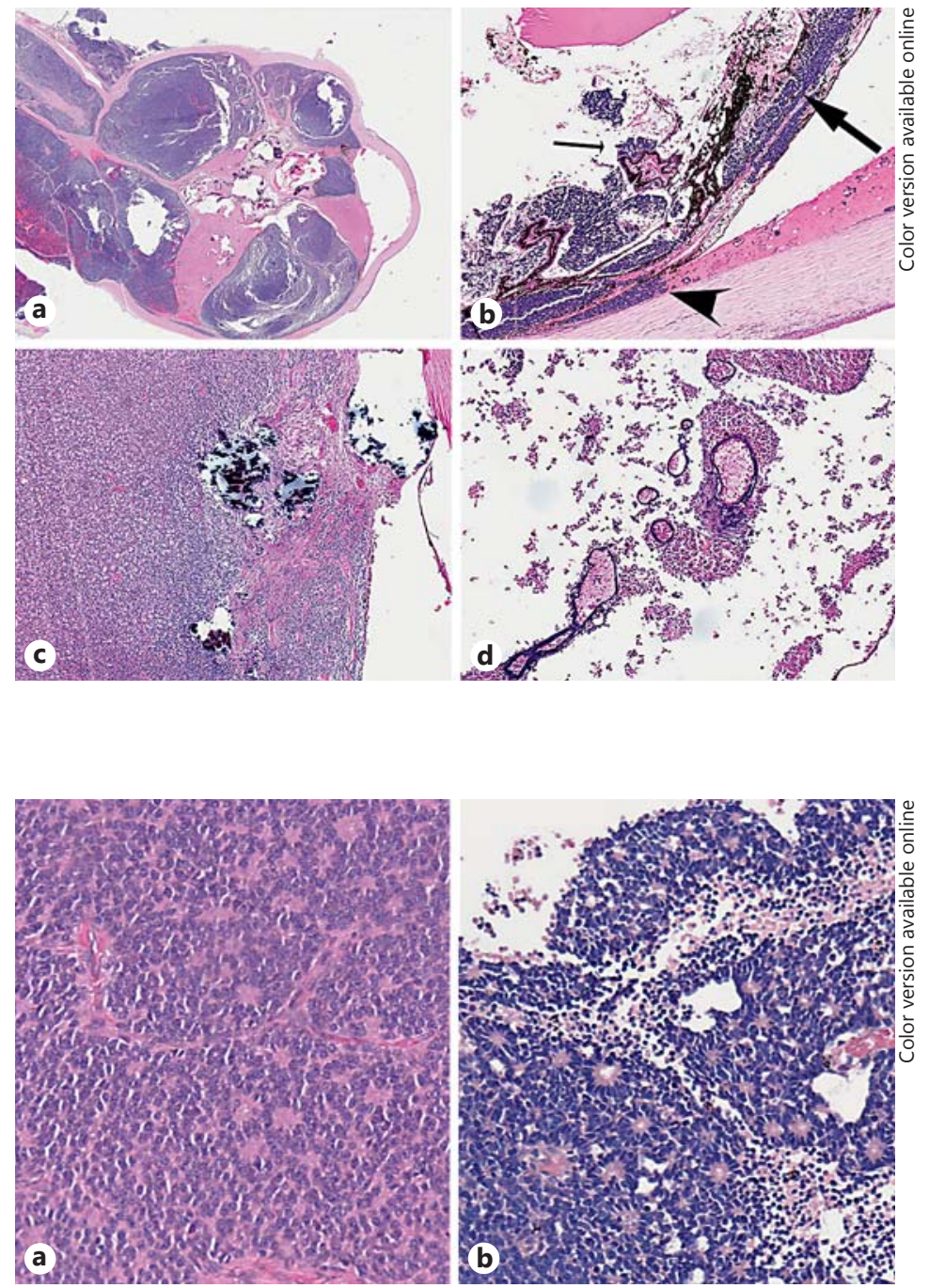
Ocular Oncology

and Pathology

\begin{tabular}{l|l}
\hline Ocul Oncol Pathol 2015;1:259-265 \\
\hline DOI: 10.1159/000381155 & $\begin{array}{l}\text { @ 2015 S. Karger AG, Basel } \\
\text { www.karger.com/oop }\end{array}$ \\
\hline
\end{tabular}

Zoroquiain et al.: Diagnosing Pathological Prognostic Factors in Retinoblastoma: Correlation between Traditional Microscopy and Digital Slides

Tumor differentiation was estimated by the presence of rosettes (fig. 4); 15 cases (31.91\%) exhibited some type of rosette, while 32 cases (68.09\%) did not have any rosettes (undifferentiated). The percentage of overall agreement was $100 \%$.

\section{Classic Morphological Features}

The growth pattern of RB cases was mixed $(n=44 ; 93.6 \%)$, showing spontaneous regression ( $\mathrm{n}=2 ; 4.3 \%)$, or endophytic $(\mathrm{n}=1 ; 2.1 \%)$. Only 2 cases had multifocal tumors (4.2\%), and necrosis was identified in 46 cases (97.9\%). An Azzopardi effect was seen in 10 cases (21.3\%; fig. 3d), while vitreal seeding was observed in 9 cases (19.2\%). All of the aforementioned features analyzed by WSI and by conventional microscopy showed an MT with $\mathrm{p}=1.00$, POA of $100 \%$, S of $100 \%$, and Sp of $100 \%$.

Calcification was seen in 45 cases using conventional microscopy (95.74\%), but using WSI, calcification was not identified in 1 case. For this particular feature, results were as follows: $\mathrm{MT}, \mathrm{p}=1.00 ; \mathrm{POA}=97.9 \% ; \mathrm{S}=97.8 \%$, and $\mathrm{Sp}=100 \%$.

\section{Discussion}

Digital pathology, and specifically WSI, has been previously implemented around the world for education [7] and research [8]. Recently, its adoption for diagnostic purposes has been increasing. It is important not to confuse WSI with telepathology systems, as they represent distinctly different technologies, mainly, static image systems, real-time systems, and WSI. Static image systems are limited by the quality of the photographs that the controller takes, while with the latter two systems, a pathologist can evaluate the entire microscopic slide and, using the software, navigate to any field of view at a wide variety of desired magnifications. The main differences between real-time systems and WSI are that the former uses a robotic microscope located at a distant site, whereas the WSI employs an automated scanner to take virtual images of the entire tissue sections, which can then be transferred to another location using web-based platforms.

In this study, we found that WSI is a valid method to detect microscopic morphological risk factors with a very good correlation between WSI findings and conventional microscopic findings (MT, $\mathrm{p}=1.00 ; \mathrm{POA}=100 \% ; \mathrm{S}=100 \%$, and $\mathrm{Sp}=100 \%$ ). As these findings could have been due to observer bias, we implemented a 'brainwash' period of 10 days between slide viewing and also reviewed the slides at random order. Studies in breast pathology have shown similar findings [9], but to the best of our knowledge, this is the first time that a comparison has been made on ocular pathology samples. It is important to emphasize that the relationship of the tumor with several anatomic structures with prognostic relevance in a small area is a characteristic of intraocular tumors.

The only feature that did not show a POA of $100 \%$ was calcification; in the 1 case where the pathologist failed to identify POA on WSI, the calcification was very small and had a fine, nodular aggregate shape. Moreover, the feature was more easily visualized based on its refringent pattern rather than on morphology alone; however, this difference was not statistically significant (MT, $\mathrm{p}=1.00$ ). This feature could be important in breast pathology when looking for microcalcifications.

In this study, only the OP sections were reviewed. It is well known that the processing of a RB includes the creation of 4 cassettes: 1 for the OP section, 1 for the margin, and 2 for the calottes. In this study, the main objective is to compare the capability of finding morphological prognostic factors in the scanned images; thus, reviewing an entire case could lead to the bias of finding a prognostic marker in different slides and record them as an agreement. This is the reason why only one slide was selected, and the one that usually has more findings is the OP section. 
Zoroquiain et al.: Diagnosing Pathological Prognostic Factors in Retinoblastoma: Correlation between Traditional Microscopy and Digital Slides

Subjectively, we found that reviewing the WSI took slightly longer than a review by microscope, but this was expected as pathologists have years in training using a microscope. By comparison, we propose that first-day medical students find maneuvering a slide via a microscope challenging, and based on their familiarity with using a mouse and computer interface, the adoption period for WSI may indeed be more rapid in this cohort. Moreover, if the software was adapted for 'touch screen' use, we believe this would make it more intuitive, thereby increasing viewing speed to either equal or surpass microscope viewing.

We found the image analyzer interface to be very user friendly (fig. $2 \mathrm{~b}$ ); we could easily asses the size of the choroidal invasion with the built-in measuring tool and annotate every important finding in order to retrieve it at a later time (e.g., for meetings or clinicopathologic rounds). Another advantage of WSI is that a global view of the specimen can be effortlessly achieved, making it easier to analyze anatomic relationships between the tumor and other structures.

The only disadvantage of WSI identified is that it does not have a condense aperture diaphragm option to highlight some membranes or calcifications; however, we had no problems detecting Bruch's membrane invasion (fig. $2 \mathrm{~b}$ ) or the presence/absence of choroidal invasion. If this proves troublesome for other membranes, periodic-acid Schiff staining can be used in these cases.

In this particular study, we found that WSI is a flexible tool which was not restricted to any specific imaging hardware. For this reason, anyone in the world with a username and password can access WSI, offering a unique opportunity for pathologists to easily collaborate and obtain second opinion consultation. Moreover, WSI is easy to understand for a nonpathologist, because it is similar to the gross specimen seen by clinicians in daily practice, thereby facilitating feedback between pathologists and clinicians, emphasizing the importance of pathology in patient diagnosis and treatment.

There are several differences between WSI and a conventional glass microscope. For instance, WSI has the obvious drawback of higher implementation costs, and it also takes time to scan the slides. Moreover, you have to become acquainted with the interface; however, for us, it only took one 6-hour session of training to feel comfortable with the software. On the other hand, WSI is very useful because it is possible to collect multiple opinions simultaneously (via viewing on a monitor) and to obtain more precise measurements of the lesion size, extent of invasion, and distance from resection margins through the use of digital analysis tools [3]. Furthermore, a remote pathologist can gain access to an expert team, not limited to ophthalmic pathologists, but even to dermatopathologists or other related pathology subspecialists, who can assist in rendering an accurate diagnosis. An accurate pathological diagnosis rendered by subspecialists is very important as an inadequate or inaccurate diagnosis can result in a gap in the ability of clinicians to treat patients.

Based on our experience of using WSI to find high-risk morphological features in RB, we think that this technology is applicable in daily use in ocular pathology; still, there are many steps that need to be completed before this becomes a reality. However, we can see how some barriers are being lifted by companies like GE Healthcare and Leica Biosystems, who have obtained a Health Canada Class II Medical Device License for creating, managing, storing, annotating, measuring, and viewing digital WSI for routine pathology use [3]. Moreover, the College of American Pathologists published guidelines to validate WSI as a diagnostic tool [1], which is a major step towards the adoption of WSI in daily pathologist practice. In these guidelines, the importance of validating the technology in each laboratory that wants to adopt this technology as a diagnostic tool is mentioned. These are major steps towards the adoption of WSI in the daily practice of pathologists.

In conclusion, WSI can be used to identify all morphological features of RB at par with conventional microscopic evaluation. It has the obvious drawback of higher initial costs, and 
it takes longer to obtain images compared to a conventional microscope. However, WSI allows for simple annotations and measurements. Also, WSI is easier to store, allows better and more rapid consultation between pathologists, and provides superior feedback for clinicians during clinicopathologic rounds because they can visualize the whole specimen as they typically see it in their daily clinical practice.

\section{Disclosure Statement}

The authors declare no conflicts of interest.

\section{References}

1 Pantanowitz L, Sinard JH, Henricks WH, et al: Validating whole slide imaging for diagnostic purposes in pathology: guideline from the College of American Pathologists Pathology and Laboratory Quality Center. Arch Pathol Lab Med 2013;137:1710-1722.

-2 Barisoni L, Nast CC, Jennette JC, et al: Digital pathology evaluation in the multicenter Nephrotic Syndrome Study Network (NEPTUNE). Clin J Am Soc Nephrol 2013;8:1449-1459.

-3 Têtu B, Evans A: Canadian licensure for the use of digital pathology for routine diagnoses: one more step toward a new era of pathology practice without borders. Arch Pathol Lab Med 2013;138:302-304.

-4 Romero Lauro G, Cable W, Lesniak A, et al: Digital pathology consultations - a new era in digital imaging, challenges and practical applications. J Digit Imaging 2013;26:668-677.

5 Mitra D, Shaw AK, Hutchings K: Trends in incidence of childhood cancer in Canada, 1992-2006. Chronic Dis Inj Can 2012;32:131-139.

6 Sastre X, Chantada GL, Doz F, et al: Proceedings of the consensus meetings from the International Retinoblastoma Staging Working Group on the pathology guidelines for the examination of enucleated eyes and evaluation of prognostic risk factors in retinoblastoma. Arch Pathol Lab Med 2009;133:1199-1202.

7 Harris T, Leaven T, Heidger P, Kreiter C, Duncan J, Dick F: Comparison of a virtual microscope laboratory to a regular microscope laboratory for teaching histology. Anat Rec 2001;265:10-14.

-8 Koch LH, Lampros JN, Delong LK, Chen SC, WoosleyJT, Hood AF: Randomized comparison of virtual microscopy and traditional glass microscopy in diagnostic accuracy among dermatology and pathology residents. Hum Pathol 2009;40:662-667.

-9 Campbell WS, Hinrichs SH, Lele SM, et al: Whole slide imaging diagnostic concordance with light microscopy for breast needle biopsies. Hum Pathol 2014;45:1713-1721. 